\title{
A NOTE ON THE DUALITY BETWEEN POISSON HOMOLOGY AND COHOMOLOGY
}

\author{
JIAFENG LÜ, XINGTING WANG AND GUANGBIN ZHUANG
}

\begin{abstract}
For a Poisson algebra $A$, by studying its universal enveloping algebra $A^{\text {pe }}$, we prove a duality theorem between Poisson homology and cohomology of $A$.
\end{abstract}

\section{INTRODUCTION}

Poisson algebras, whose deformation are intimately related to some interesting non-commutative algebras (for example, Calabi-Yau algebras), have been intensively studied recently. It is known that for a lot of Poisson algebra $A$ of dimension $\ell$, the following duality holds

$$
\operatorname{HP}_{n}(A) \cong \operatorname{HP}^{\ell-n}(A)
$$

where $\operatorname{HP}_{*}(A)$ and $\operatorname{HP}^{*}(A)$ are Poisson homology and Poisson cohomology of $A$, respectively. In this paper, we prove a criterion for the duality (1.1) to hold. In fact, for a large class of Poisson algebras $A$ of dimension $\ell$, we are able to construct a right Poisson $A$-module $\omega_{A}$ such that

$$
\operatorname{HP}_{n}\left(A, \omega_{A}\right) \cong \operatorname{HP}^{\ell-n}(A)
$$

for any $n$. See Proposition 3.6 for the details. The duality (1.2) has been observed by LaunoisRichard for a class of quadratic Poisson algebras [LR, Section 3.1]. The proof of our result will use the universal enveloping algebra $A^{p e}$ of a Poisson algebra $A$.

Throughout this paper, let $k$ denote a base field of characteristic 0. All algebras and tensor products are taken over $k$ unless otherwise stated.

\section{Universal enveloping algebras of Poisson algebras}

For a Poisson algebra $A$, one can define its universal enveloping algebra $A^{\text {pe }} \underline{\mathrm{Oh}}$. In $\mathrm{U}$, a constructive definition in terms of generators and relations are given. It is also observed, by several authors [LWZ, T, that $A^{\text {pe }}$ is canonically isomorphic to $V\left(A, \Omega_{A}\right)$, where $\Omega_{A}$ is the Kähler differential of $A$ and is equipped with the Lie-Rinehart algebra structure derived from the Poisson structure of $A$. For a detailed account of this isomorphism, one can refer to [LWZ, Proposition 5.7].

The construction of $A^{p e}$ results in an algebra map $m: A \rightarrow A^{p e}$ and a Lie map $h: A \rightarrow A^{p e}$. The map $m$ is injective and therefore we would simply consider $A$ as a subalgebra of $A^{p e}$. Also, we will write $h_{a}$ for $h(a)$.

2010 Mathematics Subject Classification. 17B63, 16E40, 16S10.

Key words and phrases. Poisson algebras, Poisson homology, Poisson cohomology, Lie-Rinehart algebras. 
For a left Poisson module $M$ with the bilinear map $\{\}:, A \otimes M \rightarrow M$, it naturally becomes left $A^{p e}$-module such that $\{a, m\}_{M}=h_{a} m$ for any $a \in A$ and $m \in M$. A similar statement holds for right Poisson modules as well. The following proposition is well known. See [U, Corollary 1].

Proposition 2.1. The category of left (resp. right) Poisson modules over a Poisson algebra $A$ is equivalent to the left (resp. right) module category over $A^{\text {pe }}$.

Clearly, $A$ can be viewed as a left $A^{p e}$-module as well as a right $A^{p e}$-module. In fact, as a left (resp. right) $A^{\text {pe }}$-module, $A$ is isomorphic to the quotient of $A^{\text {pe }}$ by the left (resp. right) ideal generated by elements $h_{a}$ where $a \in A$.

The advantage of identifying $A^{\text {pe }}$ with $V\left(A, \Omega_{A}\right)$ is that now we can use some standard results from the theory of Lie-Rinehart algebras. For example, the algebra $V\left(A, \Omega_{A}\right)$ carries a filtration which naturally passes to $A^{p e}$ via the canonical isomorphism. The filtration $\left\{F_{n}\right\}_{n \geq 0}$ is such that $A \subset F_{0} A^{p e}$ and $h_{x} \in F_{1} A^{e}$ for any $x \in A$. Now the theorem [Ri, Theorem 3.1] says the following.

Proposition 2.2. Let $A$ be a Poisson algebra. If the Kähler differential $\Omega_{A}$ is a projective A-module, then there is an A-algebra isomorphism

$$
S_{A}\left(\Omega_{A}\right) \cong \operatorname{gr}_{F} A^{p e},
$$

where $S_{A}\left(\Omega_{A}\right)$ is the symmetric A-algebra on $\Omega_{A}$.

Here are some other consequences that will play essential roles in our paper.

Let $A$ be a Poisson algebra. Consider the complex $C_{*}$ where $C_{n}=0$ for $n<0$ and $C_{n}=$ $A^{p e} \otimes_{A} \Omega_{A / k}^{n}$ for $n \geq 0$. Conventionally, we take $\Omega_{A / k}^{0}=A$. The differential $b$ is given by

$$
\begin{aligned}
b\left(a_{0} \otimes \mathrm{d} a_{1} \mathrm{~d} a_{2} \cdots \mathrm{d} a_{n}\right) & =\sum_{i=1}^{n}(-1)^{i+1} a_{0} h_{a_{i}} \otimes \mathrm{d} a_{1} \mathrm{~d} a_{2} \cdots \mathrm{d} \hat{a}_{i} \cdots \mathrm{d} a_{n} \\
& +\sum_{1 \leq i<j \leq n}(-1)^{i+j} a_{0} \otimes \mathrm{d}\left\{a_{i}, a_{j}\right\} \mathrm{d} a_{1} \cdots \mathrm{d} \hat{a}_{i} \cdots \mathrm{d} \hat{a}_{j} \cdots \mathrm{d} a_{n} .
\end{aligned}
$$

Proposition 2.3. Let $A$ be a Poisson algebra and suppose that $\Omega_{A}$ is projective over $A$. Then the complex $C_{*}$ defined above is a projective resolution of $A$ as a left $A^{\text {pe }}$-module.

Proof. This is [Ri, Lemma 4.1].

Therefore, we get the following proposition, which to our knowledge is first explicitly spelled out in $[\mathrm{H}]$.

Proposition 2.4. Let $A$ be a Poisson algebra and suppose that $\Omega_{A}$ is projective over $A$. Let $M$ be a left Poisson module and $N$ a right Poisson module. Then

$$
\operatorname{HP}^{*}(A, M) \cong \operatorname{Ext}_{A^{p e}}^{*}(A, M)
$$

and

$$
\mathrm{HP}_{*}(A, N) \cong \operatorname{Tor}_{*}^{A^{p e}}(N, A),
$$


where $\operatorname{HP}^{*}(A, M)$ is the Poisson cohomology with coefficients in $M$ and $\operatorname{HP}_{*}(A, N)$ is the Poisson homology with coefficients in $N$.

In particular, if $M=A$ (resp. $N=A)$, then $\operatorname{HP}^{*}(A, M)\left(\operatorname{resp} . \mathrm{HP}_{*}(A, N)\right)$ is simply denoted by $\mathrm{HP}^{*}(A)$ (resp. $\mathrm{HP}_{*}(A)$ ) and called the Poisson cohomology (resp. Poisson homology) of $A$. However, in light of the previous proposition, one needs not to worry too much about those definitions, at least when $\Omega_{A}$ is projective over $A$, since they are just Ext and Tor which we assume most readers have a solid understanding on.

The rest of the section will be devoted to some details on the Lie-Rinehart algebra structure on $\Omega_{A}$. First, let's recall the definition.

Definition 2.5. Let $R$ be a commutative ring with identity, $A$ a commutative $R$-algebra and $L$ a Lie algebra over $R$. The pair $(A, L)$ is called a Lie-Rinehart algebra over $R$ if $L$ is a left $A$-module and there is an anchor map $\rho: L \rightarrow \operatorname{Der}_{R}(A)$, which is an $A$-module and a Lie algebra morphism, such that the following relation is satisfied,

$$
[\xi, a \cdot \zeta]=a \cdot[\xi, \zeta]+\rho(\xi)(a) \cdot \zeta
$$

for any $a \in A$ and $\xi, \zeta \in L$.

For simplicity, we will use $\xi(a)$ for $\rho(\xi)(a)$ where $\xi \in L$ and $a \in A$. In this paper, we would always assume that $R$ is the ground field $k$. The following example is the one that we are mainly interested [H], Theorem 3.8].

Example 2.6. Let $A$ be a Poisson algebra over $k$ and $\Omega_{A}$ its Kähler differentials. Then the pair $\left(A, \Omega_{A}\right)$ becomes a Lie-Rinehart algebra over $k$ where the Lie bracket on $\Omega_{A}$ is given by

$$
[a d f, b d g]=a b d\{f, g\}+a\{f, b\} d g-b\{g, a\} d f,
$$

and the anchor map sends $d f$ to $\{f, \cdot\}$.

Now suppose that $A$ is an affine Poisson algebra and that $\Omega_{A}$ is free over $A$ of rank $\ell$ (which is equal to the Krull dimension of $A$ ) with a basis $\left\{\mathrm{d} x_{1}, \cdots, \mathrm{d} x_{\ell}\right\}$. Then for any $y \in A$, there is a unique matrix $Y=\left(Y_{i j}\right) \in M_{\ell}(A)$ such that

$$
\left[\mathrm{d} y, \mathrm{~d} x_{i}\right]=\sum_{j} Y_{i j} \mathrm{~d} x_{j}
$$

for any $i$. Define the trace of $\mathrm{d} y$ to be

$$
\operatorname{tr}(\mathrm{d} y)=\operatorname{tr} Y
$$

\section{A duality Between Poisson homology And COHOMOlogy}

In this section, we are going to prove the main result of the paper.

Lemma 3.1. Suppose that $A$ is an affine Poisson algebra and that $\Omega_{A}$ is free over $A$ of rank $\ell$. Then $\operatorname{Ext}_{A^{p e}}^{i}\left(A, A^{p e}\right)=0$ for $i \neq \ell$ and $\operatorname{Ext}_{A^{p e}}^{\ell}\left(A, A^{p e}\right) \neq 0$. 
Proof. By Proposition 2.2, gr $A^{p e} \cong A\left[y_{1}, \cdots, y_{\ell}\right]:=E$. Now we use the standard spectral sequence

$$
\operatorname{Ext}_{E}^{*}(A, E) \Rightarrow \operatorname{Ext}_{A p e}^{*}\left(A, A^{p e}\right) .
$$

Now the result follows from the fact that $\operatorname{Ext}_{E}^{i}(A, E)=0$ for $i \neq \ell$ and $\operatorname{Ext}_{E}^{\ell}(A, E) \neq 0$.

Proposition 3.2. Suppose that $A$ is an affine Poisson algebra and that $\Omega_{A}$ is free over $A$ of rank $\ell$. Let $\omega_{A}$ be the right $A^{\text {pe }}$-module $\operatorname{Ext}_{A^{p e}}^{\ell}\left(A, A^{p e}\right)$. Then for any left $A^{\text {pe }}$-module (or equivalently, left Poisson module over A) N,

$$
\operatorname{Tor}_{n}^{A^{p e}}\left(\omega_{A}, N\right) \cong \operatorname{Ext}_{A^{p e}}^{\ell-n}(A, N)
$$

Proof. This is a consequence of Ischebeck's spectral sequence and Lemma 3.1 For the details, please see [K, Theorem 1.1, Corollary 1.4].

Next we look closer at $\omega_{A}=\operatorname{Ext}_{A^{p e}}^{\ell}\left(A, A^{p e}\right)$.

Lemma 3.3. Let $A$ be as in Lemma 3.1 and let $\left\{\mathrm{d} x_{1}, \cdots, \mathrm{d} x_{\ell}\right\}$ be a free A-basis of $\Omega_{A}$. Then $\omega_{A}=\operatorname{Ext}_{A^{p e}}^{\ell}\left(A, A^{p e}\right)$ is isomorphic to $A^{\text {pe }} / J$ as right $A^{\text {pe }}$-modules, where $J$ is the right ideal generated by elements $h_{x_{i}}-\operatorname{tr}\left(\mathrm{d} x_{i}\right), i=1,2, \cdots, \ell$.

Proof. The proof is just a direct calculation. Let $C_{*}$ be the resolution as in Proposition 2.3. Then $\operatorname{Ext}_{A^{p e}}^{\ell}\left(A, A^{p e}\right)$ is the cokernel of the map

$$
\operatorname{Hom}_{A^{p e}}\left(C_{\ell-1}, A^{p e}\right) \stackrel{\partial}{\rightarrow} \operatorname{Hom}_{A^{p e}}\left(C_{\ell}, A^{p e}\right) .
$$

Notice that $C_{\ell}$ is a free left $A^{\text {pe }}$-module of rank 1 . In fact, given a free basis $\left\{\mathrm{d} x_{1}, \cdots, \mathrm{d} x_{\ell}\right\}$ of $\Omega_{A}, C_{\ell}$ is a free left $A^{p e}$-module with a basis $\left\{1 \otimes \mathrm{d} x_{1} \mathrm{~d} x_{2} \ldots \mathrm{d} x_{\ell}\right\}$. Consequently, by sending $f \in \operatorname{Hom}_{A^{p e}}\left(C_{\ell}, A^{p e}\right)$ to $f\left(1 \otimes \mathrm{d} x_{1} \mathrm{~d} x_{2} \ldots \mathrm{d} x_{\ell}\right) \in A^{p e}$, we can identify $\operatorname{Hom}_{A^{p e}}\left(C_{\ell}, A^{p e}\right)$ with $A^{p e}$ as right $A^{p e}$-modules. Now a direct calculation shows that the image of $\partial$ in (3.2) is the right ideal of $A^{\text {pe }}$ generated by elements of the form $h_{x_{i}}-\operatorname{tr}\left(\mathrm{d} x_{i}\right)$ where $i=1,2, \cdots, \ell$. This completes the proof.

Question 3.4. Is there an automorphism $\nu$ on $A^{\text {pe }}$ such that $\nu$ restricts to identity on $A$ and $\omega_{A} \cong A^{\nu}$ as right $A^{p e}$-modules?

Remark 3.5. Let everything be as in Lemma 3.3, then the canonical map $A \rightarrow A^{p e} / J=\omega_{A}$ induced by the algebra map $m: A \rightarrow A^{\text {pe }}$ is an isomorphism of right $A$-modules. This is an easy consequence of Proposition 2.2. Under this identification, the right $A^{p e}$-module structure on $\omega_{A}=A$ is given by

$$
m h_{x_{i}}=-\left\{x_{i}, m\right\}+m \operatorname{tr}\left(\mathrm{d} x_{i}\right),
$$

where $m \in A$. Or, if we think $\omega_{A}$ as a right Poisson module over $A$ with bilinear map $\{,\}_{\omega}$ : $\omega_{A} \otimes A \rightarrow \omega_{A}$, the previous equation just translate into

$$
\left\{m, x_{i}\right\}_{\omega}=-\left\{x_{i}, m\right\}+m \operatorname{tr}\left(\mathrm{d} x_{i}\right)
$$

This equation has been observed by Launois-Richard for a class of quadratic Poisson algebras [LR].

Now we are ready to deliver the main result of this note. 
Proposition 3.6. Retain the notation in Lemma 3.1 and let $\left\{\mathrm{d} x_{1}, \cdots, \mathrm{d} x_{\ell}\right\}$ be a free A-basis of $\Omega_{A}$. Then

$$
\operatorname{HP}_{n}\left(A, \omega_{A}\right) \cong \operatorname{HP}^{\ell-n}(A)
$$

for any $n$. Moreover, if $\operatorname{tr}\left(\mathrm{d} x_{i}\right)=0$ for any $i$, then

$$
\mathrm{HP}_{n}(A) \cong \operatorname{HP}^{\ell-n}(A)
$$

for any $n$.

Proof. The first statement is a consequence of Proposition 2.4 and Proposition 3.3. If $\operatorname{tr}\left(\mathrm{d} x_{i}\right)=0$ for any $i$, by Lemma $3.3 \omega_{A}$ is isomorphic to $A^{\text {pe }} / J$ where $J$ is the right ideal generated by $h_{x_{i}}$. Hence $\omega_{A}$ is isomorphic to $A$ as right $A^{p e}$-modules and therefore we have the second statement.

\section{EXAmples}

In this section, we look at some examples.

4.1. A Poisson algebra arising from a Calabi-Yau algebra. Let $A=k[x, y, z]$ with Poisson bracket given by

$$
\{z, y\}=2 x z, \quad\{z, x\}=0, \quad\{y, x\}=x^{2} .
$$

This Poisson algebra is studied by Berger-Pichereau in $\mathrm{BP}$, whose deformation gives a type of Calabi-Yau algebra. In the same paper, the Poisson homology of $A$ is also explicitly calculated $[\mathrm{BP}$, Proposition 5.7]. Clearly, the Kälher differential $\Omega_{A}$ is free over $A$ with a basis $\{\mathrm{d} x, \mathrm{~d} y, \mathrm{~d} z\}$. Also,

$$
\begin{aligned}
& {[\mathrm{d} z, \mathrm{~d} y]=\mathrm{d}\{z, y\}=2 x \mathrm{~d} z+2 z \mathrm{~d} x,} \\
& {[\mathrm{~d} z, \mathrm{~d} x]=\mathrm{d}\{z, x\}=0,} \\
& {[\mathrm{~d} y, \mathrm{~d} x]=\mathrm{d}\{y, x\}=2 x \mathrm{~d} x .}
\end{aligned}
$$

Consequently, $\operatorname{tr}(\mathrm{d} x)=\operatorname{tr}(\mathrm{d} y)=\operatorname{tr}(\mathrm{d} z)=0$ and therefore $\operatorname{HP}_{n}(A) \cong \operatorname{HP}^{3-n}(A)$. In fact, as pointed out in $[\mathrm{BP}]$, the Poisson algebra $A$ is derived from the Poisson potential $\phi=-x^{2} z$ and thus the duality is automatic.

4.2. A class of quadratic Poisson algebras. In [LR], Launois-Richard studied the following Poisson algebra $A$. As an algebra, $A$ is $k\left[X_{1}, X_{2}, \cdots, X_{\ell}\right]$ and the Poisson bracket is given by

$$
\left\{X_{i}, X_{j}\right\}=a_{i j} X_{i} X_{j}
$$

where $\left(a_{i j}\right) \in M_{n}(k)$ is an antisymmetric matrix. A direct calculation shows that

$$
\operatorname{tr}\left(\mathrm{d} X_{i}\right)=\left(\sum_{j=1}^{\ell} a_{i j}\right) X_{i}
$$

Therefore, as observed in Remark 3.5, $\omega_{A}$ is isomorphic to $A$ as right $A$-modules and the right Poisson module structure on $\omega_{A}=A$ is given by

$$
\left\{m, X_{i}\right\}_{\omega}=-\left\{X_{i}, m\right\}+\left(\sum_{j=1}^{\ell} a_{i j}\right) m X_{i}
$$


for any $m \in \omega_{A}=A$. Notice that the equation (4.4) is exactly [LR, Section 3.1(6)]. Moreover, the twisted duality $\operatorname{HP}_{n}\left(A, \omega_{A}\right) \cong \mathrm{HP}^{\ell-n}(A)$ for this particular type of Poisson algebra is given in LR, Theorem 3.4.2].

In fact, for this example, we can find an algebra automorphism $\nu$ on $A^{p e}$ such that $\left.\nu\right|_{A}=i d$ and $\omega_{A} \cong A^{\nu}$. It is the unique map given by

$$
\nu\left(X_{i}\right)=X_{i}, \quad \nu\left(h_{X_{i}}\right)=h_{X_{i}}+\left(\sum_{j=1}^{\ell} a_{i j}\right) X_{i}
$$

for any $i$.

\section{REFERENCES}

[BP] R. Berger, A. Pichereau, Calabi-Yau algebras viewed as deformations of Poisson algebras, Algebr. Represent. Theory (2013), http://dx.doi.org/10.1007/s10468-013-9417-z in press.

[H] J. Huebschmann, Poisson cohomology and quantization, J. Reine Angew. Math. 408 (1990) 57-113.

[K] Ulrich Krähmer, Poincaré duality in Hochschild (co)homology, in: New Techniques in Hopf Algebras and Graded Ring Theory, K. Vlaam. Acad. Belgie Wet. Kunsten (KVAB), Brussels, 2007, pp. 117-125.

[LR] Stéphane Launois, Lionel Richard, Twisted Poincaré duality for some quadratic Poisson algebras, Lett. Math. Phys. 79 (2) (2007) 161-174.

[LWZ] J.-F. Lü, X. Wang, G. Zhuang, Universal enveloping algebras of Poisson Hopf algebras, arXiv:1402.2007 2014.

[Oh] S.-Q. Oh, Poisson enveloping algebras, Comm. Algebra 27 (1999), 2181-2186.

[Ri] G. S. Rinehart, Differential forms on general commutative algebras, Trans. Amer. Math. Soc. 108 (1963), 195222 .

[T] Matthew Towers, Poisson and Hochschild cohomology and the semiclassical limit, arXiv:1304.6003, 2013.

[U] U. Umirbaev, Universal enveloping algebras and universal derivations of Poisson algebras, J. Algebra 354 (2012), 77-94.

Lü: Department of Mathematics, Zhejiang Normal University, Jinhua, China

E-mail address: jiafenglv@gmail.com

Wang: Department of Mathematics, University of Washington, Seattle, Washington 98195, USA

E-mail address: xingting@uw.edu

Zhuang: Department of Mathematics, University of Southern California, Los Angeles 90089-2532, USA

E-mail address: gzhuang@usc.edu 